\title{
Análisis de la Participación Académica de los y las Estudiantes con Discapacidad Sensorial de la Pontificia Universidad Católica de Chile
}

\section{Analysis of the Academic Participation of Students with Sensory Disabilities in Pontificia Universidad Católica de Chile}

\author{
Catalina García* \\ Javier Farías \\ Daniela Reyes \\ Andrea Vásquez
}

Pontificia Universidad Católica de Chile, Chile

El presente estudio buscó analizar la participación académica de estudiantes con discapacidad sensorial en las distintas áreas disciplinarias de la Pontificia Universidad Católica de Chile, así como sus facilitadores y obstaculizadores, tanto en comparación con sus compañeros/as de generación de ingreso como en la distinción entre discapacidad auditiva y visual. De este modo, se quiso aportar a la discusión respecto del rol que pueden cumplir programas de acompañamiento que promuevan una participación inclusiva y con equidad de estudiantes con discapacidad sensorial en la educación superior. Para este cometido, se utilizó un enfoque metodológico mixto, cuantitativo y cualitativo. Dentro de los resultados, destaca la mayor presencia de no vigencia en la ES y el menor rendimiento académico de estudiantes con discapacidad sensorial respecto al total de estudiantes de sus respectivos programas, sobre todo en carreras de Ciencias Naturales y Matemática, así como el reconocimiento de que la interacción de características de la trayectoria escolar, de los procesos de enseñanza y aprendizaje en educación superior y, en particular, de las áreas disciplinarias, además del tipo y grado de discapacidad, son factores facilitadores y obstaculizadores de la experiencia académica de los y las estudiantes con discapacidad sensorial de la UC.

Descriptores: Discapacidad; Estudiantes con discapacidad; Inclusión; Adecuaciones académicas; Educación superior.

The present study aimed to analyze the academic participation of students with sensory disabilities in the different disciplinary areas of the Pontificia Universidad Católica de Chile, as well as their facilitators and barriers, both in comparison with their cohort and in the distinction between hearing and visual disability. Thus, this study can become a contribution to the discussion on the role of support programs which promote inclusive and equitable participation of students with sensory disabilities in higher education. For this purpose, a mixed methodological approach, quantitative and qualitative, was used. Among the main results, the greater presence of situations of not continuing studies and the lower academic achievement of students with sensory disabilities compared to the total number of students in their respective programs, especially in Natural Sciences and Mathematics careers, as well as the acknowledgement that interaction between characteristics of their school trajectories, of the teaching and learning processes in higher education and, particularly, of the disciplinary areas, in addition to the type and degree of disability, are factors that facilitate and hinder the academic experience of students with sensory disabilities at UC.

Keywords: Disabilities; Students with disabilities; Inclusion; Academic accommodations; Higher education.

*Contacto: cigarcia@uc.cl

ISSN: 0718-7378

www.rinace.net/rlei/
Recibido: 23/11/2020

$1^{a}$ Evaluación: 14/01/2021

Aceptado: 08/04/2021 


\section{Introducción}

La educación superior (ES), constituye una oportunidad de desarrollo personal, así como para el logro de una vida plena, autónoma y de desarrollo permanente. En ese proceso, la participación de estudiantes con discapacidad (EcD) ha mostrado importantes barreras, no solo en el acceso sino en la permanencia y titulación. Si bien ha habido esfuerzos por aumentar el acceso, éstos deben ser acompañados de una comprensión inclusiva de las trayectorias universitarias y de apoyos pertinentes a dicha realidad educacional, la cual ha mostrado tener diferencias relevantes con la educación escolar, espacio que ha sido más analizado y estudiado en el marco de la participación de EcD.

Según los análisis realizados por la Organización Mundial de la Salud (OMS, 2011), cerca del $15 \%$ de la población presenta algún tipo de discapacidad. Entre los ámbitos en que se presentan más barreras de participación, destaca el educativo, especialmente en niveles superiores y, por consecuencia, en el mundo del trabajo (OMS, 2011). Hoy, se observa una creciente matrícula y participación de $\mathrm{EcD}$ en la ES durante los últimos años (AbuHamour, 2013; Adams y Holland, 2006; Higher Education Research Institute, 2011; Hewett et al., 2020; McKinney y Swartz, 2020; Milic Babic y Dowling, 2017), especialmente en países de Europa y EE.UU (Eckes y Ochoa, 2005; Higher Education Research Institute, 2011; Konur, 2006), lo que ha hecho necesario la implementación de programas y servicios de apoyo académico especializado (Bellman et al., 2015; Wessel et al., 2009). Allí estas iniciativas se enmarcan en políticas que aseguran la inclusión de las personas con discapacidad $(\mathrm{PcD})$ en diferentes niveles educativos, lo que ha favorecido el funcionamiento cada vez más homogéneo y regulado de estos programas en contextos de ES (Lissi et al., 2013).

En Latinoamérica, y específicamente en Chile, aunque se ha diversificado la cobertura de la ES durante las últimas dos décadas (Servicio de Información de Educación Superior, 2014) esta no ha sido similar entre los grupos que enfrentan más inequidades (Biggeri et al., 2019), siendo la discapacidad uno de los factores que interfiere en el acceso (Moriña et al., 2017). A nivel nacional se encuentran dos estudios. El primero (censal) mostró que el 6,6\% de las PcD accedían a la ES, (Servicio Nacional de la Discapacidad ,2004); el segundo (muestral) señala que un 9,1\% de las PcD logra completar alguna instancia de ES, mientras que el 20\% de la población sin discapacidad lo hace (Servicio Nacional de la Discapacidad, 2015).

Dados los desafíos durante la participación de EcD en la ES, este artículo se enfocó en analizar la experiencia académica de los y las EcD sensorial en las distintas áreas disciplinarias, haciendo un intento de revisar variables académicas y contextuales de manera integrada, con el fin de colaborar en la discusión respecto del rol que pueden cumplir programas de acompañamiento que promuevan una participación inclusiva en ES.

\section{Revisión de la literatura}

\subsection{Trayectoria escolar de EcD visual y auditiva en Chile}

El concepto de trayectoria académica, a modo global, se entiende como los recorridos de estudiantes respecto de estándares definidos y conceptualizados desde el sistema escolar y en donde interactúan múltiples variables contextuales e individuales (Treviño et al., 2016). Se han descrito factores individuales influyentes en las trayectorias escolares, 
siendo las NEE y la discapacidad uno de ellos (Carlberg y Granlund, 2019; Humphrey et al., 2013). Esta última se ha establecido como una variable influyente en la posibilidad de concluir la etapa escolar y, por consecuencia, en el ingreso a la ES (Sentenac et al., 2019). Dado el riesgo de deserción del sistema educativo, mencionar algunos datos de la trayectoria escolar de los estudiantes con discapacidad es relevante para establecer un continuo que contribuya a la comprensión de lo que ocurre con este grupo en educación superior, especialmente aquellos referidos a mediciones estandarizadas.

En Chile, el marco que orienta los procesos de inclusión de las personas con discapacidad lo entregan principalmente dos normas, la Convención de las Naciones Unidas sobre los Derechos de las Personas con Discapacidad (CDPD), ratificada el año 2008, y la Ley $N^{\circ} 20.422$ (Errandonea, 2016), en vigencia desde el 2010. En este sentido, una de las estrategias de apoyo para el acceso e inclusión de EcD a la educación regular, es la creación de los Programas de Integración Escolar (PIE). Aunque ha permitido mayor cobertura, se han evaluado negativamente algunos elementos como, por ejemplo, la escasa disponibilidad de materiales de estudio en braille o LSCh o el desarrollo de habilidades de escritura en español en los estudiantes sordos, situación que ha sido atribuida a que los PIE se componen mayormente de estudiantes con discapacidad de aprendizaje más que sensoriales o físicas, lo que implica ausencia de profesionales específicos (Tamayo et al., 2018).

El informe de resultados nacionales de estudiantes de $8^{\circ}$ básico en situación de discapacidad sensorial que rindieron las pruebas de Lectura y Matemática del SIMCE adaptado el año 2019, muestra que ninguno de los grupos logró superar el 50\% de respuestas correctas, revelando los bajos aprendizajes de estos estudiantes en el nivel escolar (Agencia de Calidad de la Educación, 2019). Al proyectar este escenario al ciclo de educación media, es lógico suponer que, en la medida en que los contenidos se vayan complejizando y especificando, será más difícil que los estudiantes ciegos y sordos alcancen los aprendizajes propuestos en el currículo nacional.

Respecto al ingreso de estudiantes a la universidad, las investigaciones dan cuenta de dos tipos de barreras: una donde las universidades buscan atraer especialmente a $\mathrm{EcD}$, pero sin ofrecer las condiciones para que progresen en el sistema (Milic Babic y Dowling, 2017), la otra, una discriminación en la accesibilidad a las formas de evaluación para la admisión sin ajustes (Hewett et al., 2017). Desde el año 2017 el Departamento de Evaluación, Medición y Registro Educacional (DEMRE), ha establecido procedimientos para la solicitud de adecuaciones o apoyos en la rendición de la Prueba de Selección Universitaria (PSU), ahora Prueba de Transición Universitaria. Aún con las adecuaciones, en el proceso de admisión 2020, 18\% de los postulantes ciegos y el $2 \%$ de estudiantes sordos quedaron aceptados en alguna Universidad perteneciente al SUA (DEMRE, 2020).

\subsection{Barreras para la inclusión estudiantes con discapacidad en Educación Superior}

Booth y Ainscow (2002) proponen el enfoque de barreras para el aprendizaje y la participación enfatizando que las restricciones que enfrentan las personas con discapacidad se explican por las características particulares del contexto en el cual se desenvuelven. Desde esta perspectiva, la inclusión educativa es comprendida como un proceso constante de buscar barreras en las instituciones e implementar estrategias que tiendan a su disminución en las distintas estructuras del sistema, la cultura, la política y las prácticas (Ainscow y Miles, 2008; Booth y Ainscow, 2000). Asimismo, se debe garantizar no sólo el acceso sino también la participación efectiva en actividades 
formativas, de modo que el proceso de aprendizaje se dé en condiciones de equidad (Lissi et al., 2013).

Estudios destinados a identificar las necesidades de los EcD en ES han reconocido, a nivel general, barreras en: (1) la infraestructura que interfiere en el acceso a los espacios físicos e implementación de ajustes curriculares (Borland y James, 1999; Mella et al., 2014); (2) actitudes y creencias sobre la capacidad (Konur, 2006; Moriña et al., 2015; Rodríguez y Álvarez, 2014; Salinas et al., 2013) y la compatibilidad de las NEE asociadas a discapacidad con ciertas áreas disciplinarias, lo que se traduce en la creencia de que sólo algunos pueden estudiar ciertas carreras, dependiendo de su tipo-grado de discapacidad, afectando la creación de ajustes para que su participación sea posible (Biggeri et al., 2019; Moriña et al., 2017). Por ejemplo, la accesibilidad a carreras de índole científica y práctica es escasa, y las elecciones del estudiante se basan en la evaluación de las condiciones de acceso y las disposiciones actitudinales (Milic Babic y Dowling, 2017); (3) la falta de formación de los docentes (Díez y Sánchez, 2015; Lombardi et al., 2015; Medrano, 2009); y (4) la existencia de un currículo rígido con pocas posibilidades de adaptarse a la diversidad de necesidades educativas existentes, lo que genera dificultades en el acceso a los materiales y la falta de aplicación del diseño universal para la enseñanza a la práctica docente (Díez y Sánchez, 2015).

En relación al último tipo de barrera mencionada se generan necesidades específicas que requieren de la puesta en marcha de acciones de capacitación docente, apoyos académicos específicos y acompañamiento estudiantil para disminuirla. Las primeras derivan en necesidades específicas que, en el caso de estudiantes ciegos o con baja visión, se traducen en la utilización de formato braille o digitales compatibles con lectores de pantalla. Los estudiantes con baja visión necesitan documentos editables para configurarlos en tipo de letra, tamaño o contraste. Para el trabajo con contenidos matemáticos y científicos (fórmulas y expresiones), los elementos deben ser presentados en formatos compatibles con lectores de pantalla o en complemento con editores específicos que permitan su lectura a través de los sintetizadores de voz y también su salida en línea braille. En cuanto a contenido gráfico, se deben incorporar descripciones de texto alternativo y/o ser confeccionado con material táctil. Las personas hipoacúsicas se benefician de enseñanza que ocupa elementos visuales, como información por escrito, mapas conceptuales, destacado de elementos, etc. (Erbas, 2017; Domínguez y Velasco, s.f.; Hayes, s.f.). Además, es importante hablar mirando de frente, respetar los turnos de conversación, parafrasear o repetir información e incorporar expresiones faciales y de subtítulos. Los estudiantes sordos requieren del complemento visual, aun cuando lo fundamental es la mediación de un Intérprete de Lengua de Señas Chilena (LSCh) para acceder y comprender los contenidos e interactuar con la comunidad oyente. Además, requerirán apoyo para desarrollar habilidades de escritura en español, debido a que gran parte de la información se encuentra disponible en esta modalidad.

\subsection{Educación superior, discapacidad y trayectorias académicas}

\subsubsection{Permanencia de estudiantes con discapacidad en educación superior}

En Latinoamérica existen pocos registros respecto de la permanencia de EcD en ES, no obstante, puede expresarse con cierto grado de certeza que los EcD representan un colectivo próximo al 1\% de la población universitaria total (Galloni et al., 2011). En España, el panorama es similar. El 2018 se aplicó el IV Estudio sobre el grado de inclusión del sistema universitario español, dando cuenta de una representación del 1,5\% 
(Fundación Universia, 2019). Un porcentaje bajo en comparación con el de otros países como Canadá, donde este se mueve entre un $5 \%$ y un $10 \%$ del total del estudiantado universitario. Con relación a la distribución por áreas disciplinarias, el 54\% cursan grados de Ciencias Sociales y Jurídicas, el 26\% de Ciencias, Tecnología, Ingeniería y Matemáticas y el $19,5 \%$ de Artes y Humanidades.

A nivel nacional no se cuenta con datos actualizados sobre la permanencia de estudiantes con discapacidad en la ES, pues no existe regulación central sobre la materia, ya que a diferencia de otros países donde el Ministerio de Educación tiene más participación en el tema, en Chile es el SENADIS quien da algunas orientaciones y opciones de postulaciones a financiamiento (Lissi et al., 2013). A pesar de que la Ley N ${ }^{\circ} 20.422$ de 10 de febrero que establece normas sobre igualdad de oportunidades e inclusión social de personas con discapacidad define responsabilidades para facilitar el acceso de EcD a la ES, así como en ajustes al material de estudio y medios de enseñanza, no se especifican estándares ni acciones que orienten cómo poner en marcha estos mecanismos, ni formas de articulación para favorecer la transición entre el nivel escolar y superior; tampoco existe pronunciamiento respecto al financiamiento ni a indicaciones como ajustes curriculares, trayectorias distintas o apoyos específicos. En ocasiones los lineamientos de esta ley no pueden operar en concordancia con otras leyes o normativas, por ejemplo, una trayectoria diferenciada en tiempos genera dificultades para mantener la gratuidad, que permite financiamiento de la carrera, pero en el tiempo oficial de duración de ésta. A su vez, las diversas barreras asociadas al acceso físico, virtual o al material de estudio llevan a que los EcD tarden más en terminar sus carreras (Paz-Maldonado, 2020). Dado esto, las IES entregan una respuesta educativa de naturaleza diversa, a voluntad y criterio de cada una de ellas.

Se puede concluir que para generar adecuadas oportunidades de acceso, permanencia y egreso de EcD en la Universidad se requiere de: (a) un marco legal claro que impida la discriminación; (b) una entidad central que se ocupe de informar y apoyar a las instituciones y a los alumnos; (c) algún sistema de becas y de asignación de recursos para los alumnos; (d) consensuar prácticas y líneas de acción base entre los programas de apoyo; y (e) coordinación y asesoría entre el programa de apoyo y los docentes (Rodríguez y Valenzuela, 2019; Zuzulich et al., 2014).

\subsubsection{Políticas y estrategias de apoyo en laUC}

A lo largo de su historia, la UC se ha comprometido con ser un aporte en la disminución de brechas e inequidades en distintos ámbitos. Uno de los ejes ha sido favorecer el ingreso (desde 1991) y la permanencia y egreso de estudiantes con NEE mediante distintas acciones, destacándose la creación del PIANE, en el 2006. A partir del año 2017 pasa a formar parte de la Dirección de Inclusión, unidad creada el año 2014 al alero de la Vicerrectoría Académica.

La orientación del Programa es primordialmente académica y cumple una labor de asesor, es de participación voluntaria y su estrategia se compone de cuatro elementos: implementación de adecuaciones curriculares no significativas; acompañamiento a las Unidades Académicas y los estudiantes; apoyos académicos específicos y trabajo con la comunidad UC. Los apoyos específicos se otorgan de manera institucional y formal, a partir del año 2016 (y, por tanto, hay registro sistemático), complementando así los apoyos individuales financiados por SENADIS. Desde ese año, para entregar los apoyos se priorizan los recursos disponibles a partir de una evaluación de la pertinencia de la 
solicitud ${ }^{1}$ por parte del equipo PIANE. Esta es la unidad encargada de evaluar y definir los ajustes generales, informados semestralmente a docentes, para luego ofrecer instancias de asesoría y capacitación. Aun cuando existan adecuaciones curriculares, en ocasiones las necesidades de los estudiantes pueden ser más específicas debido a dificultades para acceder a la información o brechas académicas asociadas a trayectorias escolares. A ello se suman desafíos relacionados con el desarrollo de competencias para la vida universitaria, así como para la vida laboral.

La participación de PcD en ES, presenta diversas barreras que deben ser abordadas con el fin de potenciar las oportunidades de aprendizaje profundo y con ello las posibilidades de continuar un proceso de desarrollo óptimo que incluya opciones de inserción laboral plena y con equidad. Por consiguiente, el presente artículo responde al siguiente objetivo:

Analizar la participación académica de los y las estudiantes con discapacidad sensorial en las distintas áreas disciplinarias de la Pontificia Universidad Católica de Chile, así como sus facilitadores y obstaculizadores, tanto en comparación con sus pares de generación de ingreso como en la distinción entre discapacidad auditiva y visual.

\section{Método}

Se utilizó un enfoque metodológico cuantitativo con el objeto de cuantificar, integrar y consolidar los datos relativos a participación, situación y rendimiento académicos, tenencia y tipo de apoyos permanentes de los y las EcD sensorial de la UC. Las variables cuantitativas utilizadas fueron las siguientes:

- Discapacidad sensorial (dummy): indica presencia (Sí) o ausencia (No) de discapacidad sensorial de los y las estudiantes analizados.

- Clasificación carreras College: agrupación de programas cursados por estudiantes de la UC. Categorías: Arte y Humanidades (AH), Ciencias Naturales y Matemática (CNM) y Ciencias Sociales (CS).

- Situación académica: corresponde al estado de vigencia de un estudiante en un determinado programa de la UC a agosto 2020. Categorías: Finalización (completa un programa de ciclo inicial o terminal), No Vigencia (renuncia, abandona, es eliminado/a o declarado/a no vigente por la comisión de permanencia de la universidad respecto a un programa de la UC) y Vigencia (está en progreso o suspendido temporalmente en un programa de la UC).

- Porcentaje de créditos aprobados inscritos de un estudiante vigente en un programa de la UC. Es un indicador del rendimiento académico.

- Tipo de discapacidad sensorial: auditiva o visual.

- Tenencia de apoyo permanente (dummy): indica presencia (Sí) o ausencia (No) de apoyos permanentes brindados por PIANE a cada estudiante con discapacidad sensorial en el período 2016-2020.

\footnotetext{
${ }^{1}$ En caso de considerarse no pertinente el apoyo específico solicitado, se apoya al estudiante con otras redes y servicios de la Universidad.
} 
- Tipo de apoyo permanente: distingue categorías de apoyos permanentes. Categorías: Académicos (tutorías), Accesibilidad (ayudante ejecutor, tomador de apuntes, intérprete LSCh y/o transcriptor) y Ambos (académicos y de accesibilidad).

Respecto a la muestra y muestreo, el estudio consideró de manera censal a 27.972 estudiantes que han cursado algún programa de la UC siendo o teniendo como par de generación de ingreso a EcD sensorial entre los años 1983 y 2020. Por su parte, los instrumentos de obtención de información cuantitativos utilizados fueron bases de datos institucionales. El trabajo de campo cuantitativo consistió en la integración y consolidación de una base de datos única, entre agosto y octubre 2020. El análisis de datos cuantitativo fue de corte descriptivo, considerando medias y proporciones. Finalmente, respecto a los procedimientos de resguardos éticos, se tomó la decisión de que la recolección, procesamiento y análisis fuese trabajada sólo por uno de las personas autores del artículo, quien fue autorizada para este fin específico, con el objeto de cautelar por la confidencialidad, anonimato y privacidad de los y las estudiantes analizados.

El enfoque cualitativo buscó integrar las percepciones de representantes de dirección, docencia o asuntos estudiantiles de carreras de la universidad y de ejecutores de apoyos permanentes por parte de PIANE respecto a los facilitadores y obstaculizadores del aprendizaje de EcD sensorial en la UC, siguiendo el objetivo del estudio. Las categorías de análisis cualitativo utilizadas fueron las siguientes: (a) experiencia o trayectoria escolar;

(b) tipo de discapacidad, ya sea visual o auditiva; y (c) facilitadores y barreras de la experiencia académica universitaria. Se optó por utilizar estas categorías de análisis, en consonancia con el objetivo del artículo y la revisión de literatura realizada, ya que resumen los grandes factores que pueden explicar la situación académica de los y las estudiantes con EcD en la universidad. Dentro de los y las participantes, se contó con la participación de 7 académicos y/o profesionales en cargos de dirección, docencia o acompañamiento estudiantil, pertenecientes a programas de apoyo específicos y carreras (en adelante, APC); y 1 profesional y 2 tutores pares con labores de apoyo académico (en adelante, APA) quienes fueron convocados en razón de su experiencia de trabajo con EcD y considerando la variedad de roles, programas y carreras en las que se desempeñan.

Los instrumentos de obtención de información cualitativos fueron dos grupos focales, uno compuesto por el grupo APC y otro por el grupo APA, a fin de profundizar en facilitadores u obstaculizadores de la experiencia académica de los y las EcD sensorial en la UC.

El trabajo de campo cualitativo consistió en la elaboración de una pauta de grupo focal y en la ejecución de estos durante el mes de septiembre de 2020. El análisis de datos cualitativo siguió los planteamientos del análisis de contenido y se materializó, en primer lugar, en un análisis descriptivo y, luego, en un análisis relacional de los grupos focales. Finalmente, respecto a los procedimientos de resguardos éticos, cada participante de los grupos focales leyó y firmó un consentimiento informado, por el cual aceptó participar voluntariamente en el estudio, garantizando la confidencialidad y el uso exclusivo de las declaraciones emitidas para este fin. 


\section{Resultados}

\subsection{Análisis cuantitativo}

A continuación, se presentan datos relativos a la participación, situación y rendimiento académicos de estudiantes con y sin discapacidad sensorial en las carreras ligadas a las Artes y Humanidades, Ciencias Naturales y Matemática y Ciencias Sociales.

\section{Cuadro 1}

Participación de estudiantes, según presencia o ausencia de discapacidad sensorial y clasificación de carrera cursada. Período 1983-2020

\begin{tabular}{|c|c|c|c|c|c|}
\hline \multirow{3}{*}{ ClaSifiCACIÓN DE CARRERAS COLLEGE } & \multicolumn{5}{|c|}{ DISCAPACIDADSENSORIAL } \\
\hline & \multicolumn{2}{|c|}{ No } & \multicolumn{2}{|c|}{$\underline{\mathbf{S}} \mathbf{i}$} & Total \\
\hline & $\mathbf{N}$ & $\%$ & $\mathbf{N}$ & $\%$ & $\mathbf{N}$ \\
\hline Total & 27747 & $99 \%$ & 225 & $1 \%$ & 27972 \\
\hline Artes y Humanidades & 1811 & $98 \%$ & 36 & $2 \%$ & 1847 \\
\hline Ciencias Naturales y Matemática & 11072 & $99 \%$ & 57 & $1 \%$ & 11129 \\
\hline Ciencias Sociales & 14864 & $99 \%$ & 132 & $1 \%$ & 14996 \\
\hline
\end{tabular}

Según el Cuadro 1, 27.972 estudiantes han cursado algún programa de la UC siendo o teniendo como par de generación de ingreso a estudiantes con discapacidad sensorial entre los años 1983 y 2020. De ellos, sólo el 1\% (225) tiene alguna discapacidad sensorial, proporción que prácticamente no varía si se compara por la clasificación de carreras de College.

\section{Cuadro 2}

Situación académica de estudiantes según presencia o ausencia de discapacidad sensorial y clasificación de carrera cursada. Período 1983-2020

\begin{tabular}{|c|c|c|c|c|c|c|c|c|c|c|c|c|}
\hline & \multicolumn{3}{|c|}{$\begin{array}{c}\text { Artes y } \\
\text { Humanidades }\end{array}$} & \multicolumn{3}{|c|}{$\begin{array}{c}\text { CCNN y } \\
\text { Matemática }\end{array}$} & \multicolumn{3}{|c|}{ CCSS } & \multicolumn{3}{|c|}{ Total } \\
\hline & $\mathbf{N}$ & $\%$ & $\mathbf{D}$ & $\mathbf{N}$ & $\%$ & D & $\mathbf{N}$ & $\%$ & D & $\mathbf{N}$ & $\%$ & D \\
\hline Finalización & 612 & 33 & \multirow{3}{*}{$\begin{array}{l}+14 \\
\text { pp. }\end{array}$} & 2109 & 19 & & 6638 & 44 & \multirow{3}{*}{$\begin{array}{l}+1 \\
\text { pp. }\end{array}$} & 9359 & 33 & \multirow{3}{*}{$\begin{array}{l}+7 \\
\mathrm{pp}\end{array}$} \\
\hline No & 595 & 33 & & 2096 & 19 & +4 & 6579 & 44 & & 9270 & 33 & \\
\hline Sí & 17 & 47 & & 13 & 23 & pp. & 59 & 45 & & 89 & 40 & \\
\hline No vigencia & 488 & 26 & \multirow{3}{*}{$\begin{array}{l}-9 \\
\text { n }\end{array}$} & 1677 & 15 & & 2120 & 14 & \multirow{3}{*}{$\begin{array}{l}+4 \\
\text { pp. }\end{array}$} & 4285 & 15 & \multirow{3}{*}{$\begin{array}{l}+5 \\
\mathrm{pp}\end{array}$} \\
\hline No & 482 & 27 & & 1661 & 15 & +13 & 2096 & 14 & & 4239 & 15 & \\
\hline Sí & 6 & 17 & & 16 & 28 & pp. & 24 & 18 & & 46 & 20 & \\
\hline Vigencia & 747 & 40 & \multirow{3}{*}{$\begin{array}{l}-4 \\
\text { pp. }\end{array}$} & 7343 & 66 & & 6238 & 42 & \multirow{3}{*}{$\begin{array}{l}-5 \\
\mathrm{pp}\end{array}$} & 14328 & 51 & \multirow{3}{*}{$\begin{array}{l}-11 \\
\text { pp. }\end{array}$} \\
\hline No & 734 & 41 & & 7315 & 66 & -17 & 6189 & 42 & & 14238 & 51 & \\
\hline Sí & 13 & 36 & & 28 & 49 & pp. & 49 & 37 & & 90 & 40 & \\
\hline
\end{tabular}

Nota. * Dif. Sí-Total.

Según el Cuadro 2, el 15\% de la población de estudio no finalizó sus estudios, es decir, se encuentran en estado de no vigencia. Esta proporción no varía si se observan las Ciencias Sociales y Ciencias Naturales y Matemática, sin embargo, la no vigencia aumenta en 11 puntos porcentuales en las Artes y Humanidades.

Por su parte, la no vigencia de los y las estudiantes con discapacidad sensorial asciende al $20 \%$ para el total de las carreras. Esta proporción disminuye levemente en las Ciencias Sociales y Artes y Humanidades, 2 y 3 puntos porcentuales, respectivamente, y aumenta 
8 puntos porcentuales en las Ciencias Naturales y Matemática, respecto al total de carreras.

Por otro lado, es preciso mencionar que el 33\% de la población de estudio ha finalizado al menos un programa de estudios. Esta proporción se mantiene para las Artes y Humanidades, aumenta 11 puntos porcentuales para las Ciencias Sociales y disminuye 14 puntos porcentuales en las Ciencias Naturales y Matemática, respecto al total de carreras.

Por su parte, la finalización de al menos un programa de estudio de los y las estudiantes con discapacidad sensorial asciende al $40 \%$ para el total de las carreras. Esta proporción aumenta en las Ciencias Sociales y Artes y Humanidades - 5 y 7 puntos porcentuales, respectivamente -, y disminuye 17 puntos porcentuales en las Ciencias Naturales y Matemática, respecto al total de carreras.

\section{Figura 1}

Media de porcentaje de créditos aprobados inscritos de estudiantes vigentes, según clasificación de carrera cursada

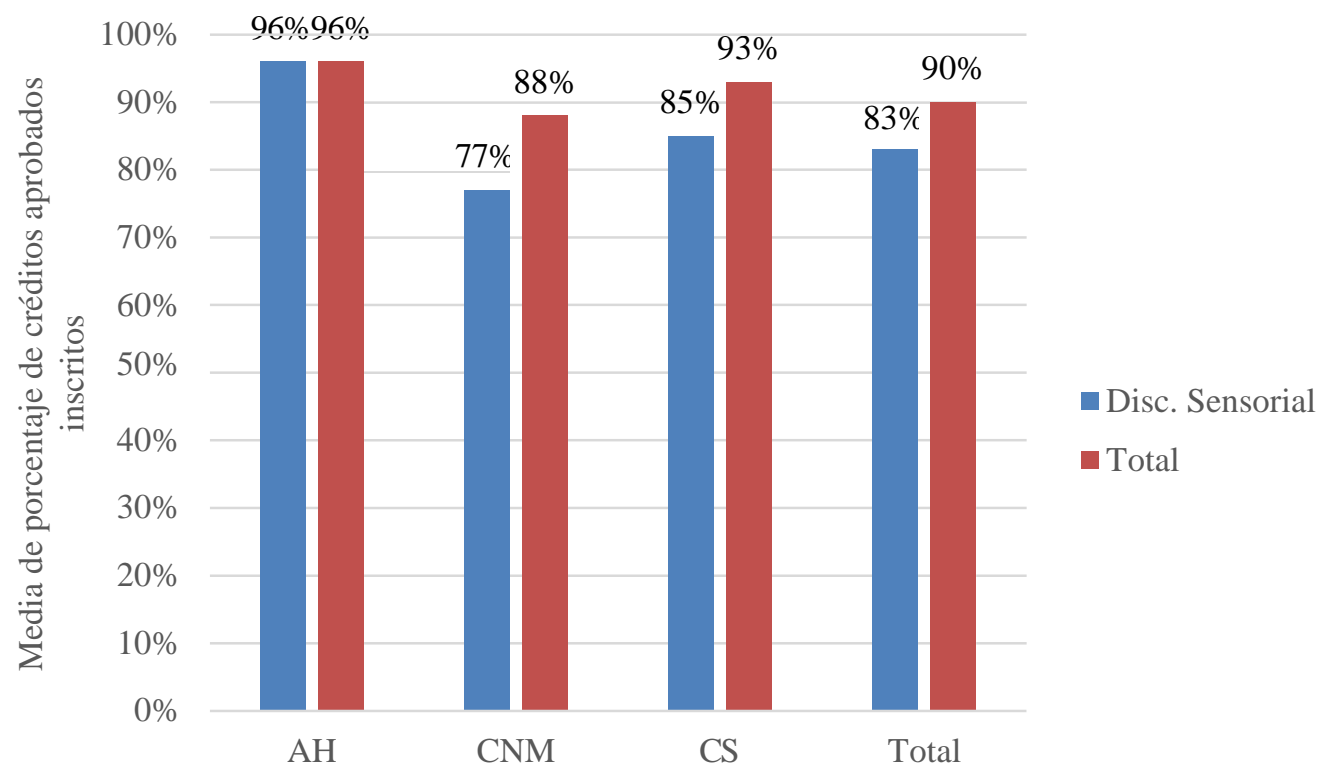

Clasificación Carreras College

Según la Figura 1, los y las estudiantes vigentes en algún programa de la UC, siendo o teniendo como par de generación de ingreso a estudiantes con alguna discapacidad sensorial, aprueban el $90 \%$ de sus créditos inscritos en promedio. Esta proporción aumenta en las Ciencias Sociales y Artes y Humanidades -3 y 6 puntos porcentuales, respectivamente- y disminuye 2 puntos porcentuales en las Ciencias Naturales y Matemática, respecto al total de carreras.

Por su parte, los y las estudiantes con discapacidad sensorial aprueban el $83 \%$ de sus créditos inscritos en promedio para el total de las carreras. Esta proporción aumenta en las Ciencias Sociales y Artes y Humanidades (2 y 13 puntos porcentuales, respectivamente), y disminuye 6 puntos porcentuales en las Ciencias Naturales y Matemática, respecto al total de carreras.

A continuación, se presentan datos relativos a la tenencia y el tipo de apoyos permanentes de estudiantes con discapacidad sensorial, según clasificación de carreras de College. 


\section{Cuadro 3}

Tenencia de apoyos permanentes, según tipo de discapacidad sensorial y clasificación de carrera cursada. Período 2016-2020

\begin{tabular}{|c|c|c|c|c|c|c|c|c|}
\hline & \multicolumn{2}{|c|}{ Artes y Humanidades } & \multicolumn{2}{|c|}{$\begin{array}{c}\text { CCNN y } \\
\text { Matemática }\end{array}$} & \multicolumn{2}{|c|}{ CCSS } & \multicolumn{2}{|c|}{ Total } \\
\hline & $\mathbf{N}$ & $\%$ & $\mathbf{N}$ & $\%$ & $\mathbf{N}$ & $\%$ & $\mathbf{N}$ & $\%$ \\
\hline Total & \multicolumn{2}{|c|}{17} & \multicolumn{2}{|c|}{40} & \multicolumn{2}{|c|}{64} & \multicolumn{2}{|c|}{121} \\
\hline No & 8 & $47 \%$ & 17 & $43 \%$ & 24 & $38 \%$ & 49 & $40 \%$ \\
\hline Sí & 9 & $53 \%$ & 23 & $58 \%$ & 40 & $53 \%$ & 72 & $60 \%$ \\
\hline Auditiva & \multicolumn{2}{|c|}{9} & \multicolumn{2}{|c|}{26} & \multicolumn{2}{|c|}{25} & \multicolumn{2}{|c|}{60} \\
\hline No & 5 & $56 \%$ & 8 & $31 \%$ & 12 & $48 \%$ & 25 & $42 \%$ \\
\hline Sí & 4 & $44 \%$ & 18 & $69 \%$ & 13 & $52 \%$ & 35 & $58 \%$ \\
\hline Visual & \multicolumn{2}{|c|}{8} & \multicolumn{2}{|c|}{14} & \multicolumn{2}{|c|}{39} & \multicolumn{2}{|c|}{61} \\
\hline No & 3 & $38 \%$ & 9 & $64 \%$ & 12 & $31 \%$ & 24 & $39 \%$ \\
\hline Sí & 5 & $63 \%$ & 5 & $36 \%$ & 27 & $59 \%$ & 37 & $61 \%$ \\
\hline
\end{tabular}

Según el Cuadro 3, el 60\% de los y las 121 estudiantes con discapacidad sensorial regulares en un programa de la UC entre los años 2016 y 2020 han tenido algún apoyo permanente por parte de PIANE. Esta proporción disminuye en las Artes y Humanidades y Ciencias Naturales y Matemática (7 y 2 puntos porcentuales, respectivamente), y aumenta 3 puntos porcentuales en las Ciencias Sociales, respecto al total de carreras.

Por su parte, el 58\% de los y las estudiantes con discapacidad auditiva regulares entre los años 2016 y 2020 han tenido algún apoyo permanente por parte de PIANE. Esta proporción disminuye en las Ciencias Sociales y Artes y Humanidades (6 y 14 puntos porcentuales, respectivamente), y aumenta 11 puntos porcentuales en las Ciencias Naturales y Matemática, respecto al total de carreras.

Finalmente, el $61 \%$ de los y las estudiantes con discapacidad visual regulares entre los años 2016 y 2020 han tenido algún apoyo permanente por parte de PIANE. Esta proporción aumenta en las Ciencias Sociales y Artes y Humanidades (8 y 2 puntos porcentuales, respectivamente), y disminuye 25 puntos porcentuales en las Ciencias Naturales y Matemática, respecto al total de carreras.

Según el Cuadro 4, de los y las 72 estudiantes con discapacidad sensorial que han tenido algún apoyo permanente por parte de PIANE entre los años 2016 y 2020, el $42 \%$ ha tenido apoyos académicos, el 19\% apoyos ligados a la accesibilidad para la información y producción y el 39\% de ambos tipos. Esta distribución (de mayor a menor proporción: académicos, ambos y accesibilidad) se mantiene en las Ciencias Sociales, y varía para las Artes y Humanidades y Ciencias Naturales y Matemática, siendo mayores los apoyos de ambos tipos, luego académicos y después de accesibilidad, para ambos tipos decarreras.

Por su parte, de los y las 35 estudiantes con discapacidad auditiva que han tenido algún apoyo permanente por parte de PIANE entre los años 2016 y 2020, el 23\% ha tenido sólo apoyos académicos, el 29\% sólo apoyos ligados a la accesibilidad para la información y producción y el $49 \%$ de ambos tipos. Esta distribución (de mayor a menor proporción: ambos, accesibilidad y académicos) se mantiene para todos los tipos de carreras.

Finalmente, de los y las 37 estudiantes con discapacidad visual que han tenido algún apoyo permanente por parte de PIANE entre los años 2016 y 2020, el 59\% ha tenido sólo apoyos académicos, el $11 \%$ sólo apoyos ligados a la accesibilidad para la información y producción y el $30 \%$ de ambos tipos. Esta distribución (de mayor a menor proporción: académicos, ambos y accesibilidad) se mantiene para todos los tipos de carreras. 


\section{Cuadro 4}

Tipo de apoyos permanentes, según tipo de discapacidad sensorial y clasificación de carrera cursada. Período 2016-2020

\begin{tabular}{|c|c|c|c|c|c|c|c|c|}
\hline & \multicolumn{2}{|c|}{$\begin{array}{c}\text { Artes y } \\
\text { Humanidades }\end{array}$} & \multicolumn{2}{|c|}{$\begin{array}{c}\text { CCNN y } \\
\text { Matemática }\end{array}$} & \multicolumn{2}{|c|}{ CCSS } & \multicolumn{2}{|c|}{ Total } \\
\hline & $\mathbf{N}$ & $\%$ & $\mathbf{N}$ & $\%$ & $\mathbf{N}$ & $\%$ & $\mathbf{N}$ & $\%$ \\
\hline Total & \multicolumn{2}{|c|}{9} & \multicolumn{2}{|c|}{23} & \multicolumn{2}{|c|}{40} & \multicolumn{2}{|c|}{72} \\
\hline Académico & 3 & $33 \%$ & 7 & $30 \%$ & 20 & $50 \%$ & 30 & $42 \%$ \\
\hline Accesibilidad & 2 & $22 \%$ & 7 & $30 \%$ & 5 & $13 \%$ & 14 & $19 \%$ \\
\hline Ambos & 4 & $44 \%$ & 9 & $39 \%$ & 15 & $38 \%$ & 28 & $39 \%$ \\
\hline Auditiva & \multicolumn{2}{|c|}{4} & \multicolumn{2}{|c|}{18} & \multicolumn{2}{|c|}{13} & \multicolumn{2}{|c|}{35} \\
\hline Académico & 0 & $0 \%$ & 3 & $23 \%$ & 3 & $23 \%$ & 8 & $23 \%$ \\
\hline Accesibilidad & 1 & $25 \%$ & 3 & $23 \%$ & 3 & $23 \%$ & 10 & $29 \%$ \\
\hline Ambos & 3 & $75 \%$ & 7 & $54 \%$ & 7 & $54 \%$ & 17 & $49 \%$ \\
\hline Visual & \multicolumn{2}{|c|}{5} & \multicolumn{2}{|c|}{5} & \multicolumn{2}{|c|}{27} & \multicolumn{2}{|c|}{37} \\
\hline Académico & 3 & $60 \%$ & 2 & $40 \%$ & 17 & $63 \%$ & 22 & $59 \%$ \\
\hline Accesibilidad & 1 & $20 \%$ & 1 & $20 \%$ & 2 & $7 \%$ & 4 & $11 \%$ \\
\hline Ambos & 1 & $20 \%$ & 2 & $40 \%$ & 8 & $30 \%$ & 11 & $30 \%$ \\
\hline
\end{tabular}

\subsection{Análisis cualitativo}

Se presenta un análisis descriptivo y un análisis relacional a partir del cual se propone un esquema que busca contribuir a la comprensión del fenómeno.

\subsubsection{Análisis descriptivo}

Se identificaron tres dimensiones que aportan en la comprensión de la experiencia de EcD sensorial en ES. Desde la perspectiva de APC estas son: (a) las características de la trayectoria escolar; y desde la perspectiva de ambos grupos (b) el tipo y grado de discapacidad y (c) facilitadores y barreras de la experiencia académica universitaria.

APC manifiesta su preocupación por los escasos ingresos de EcD a las carreras que representan, atribuyendo este fenómeno a la baja tendencia nacional de ingreso de este grupo a la ES. Las características de la educación escolar se reconocen como un factor influyente en el escaso abordaje de contenidos mínimos o en desarrollo de competencias transversales, existiendo una valoración negativa de esta etapa debido a las inequidades asociadas a la falta de metodologías accesibles en la enseñanza de contenidos.

$$
\begin{aligned}
& \text {...ha habido un aprendizaje inequitativo, por menos condiciones de acceso ... pasa a } \\
& \text { no ser brecha, si no que conocimientos inexistentes...no había ningún profesor } \\
& \text { especialista, que pudiese hacer la transformación de ese material en Braille, o bien no } \\
& \text { contaban con un intérprete de LSCh en la sala... se hace más evidente en algunas } \\
& \text { áreas. (60, APC-6) }
\end{aligned}
$$

El área de CNM se reconoce como menos desarrollada en la educación escolar general, acentuándose en $\mathrm{EcD}$ sensorial debido a las barreras para acceder a los contenidos.

$$
\begin{aligned}
& \text {...en los colegios no se recibe la mejor formación en términos del área de las } \\
& \text { matemáticas y las ciencias, pero además es muy probable que en estudiantes con alguna } \\
& \text { discapacidad sensorial...la brecha es mucho más alta. (64, APC-4) }
\end{aligned}
$$

Las características de la discapacidad serían otra variable que diferencia la profundidad de las brechas específicas. Tanto APC como APA, distinguen que EcD visual enfrentan barreras relativas a la accesibilidad de contenidos, especialmente en el área de CNM, mientras que en EcD auditiva, y específicamente estudiantes sordos, las barreras de acceso a la información en su lengua natural suelen afectar de modo más transversal el 
aprendizaje. Sumado a lo anterior, las estrategias de apoyo focalizado para este grupo parecen encontrarse menos definidas.

...con estudiantes sordas, lo que más nos costó fue, fueron los cursos del área de las ciencias, por un tema como de la brecha ...son de contenidos bien abstractos. (100, APC-4)

...no nos hemos adaptado...estamos al debe con la enseñanza de ramos que son de ciencia, de ciencias naturales y matemáticas. (46, APC-5)

...tfs el tema de la lectoescritura del español, que si bien tenemos apoyos...no existe un apoyo específico para los alumnos con sordera ...podría ser una necesidad que en este momento no está cubierta. (14,APA-1)

La posibilidad de que algunos de los casos puedan significar mayores desafíos se presenta como una importante preocupación, especialmente cuando las carreras u otras unidades de apoyo implementaron estrategias que impactaron someramente en los resultados académicos de los estudiantes o en donde tardaron en mostrar algún efecto. Pese a lo anterior, cuando estas experiencias terminaron por favorecer el aprendizaje, aun cuando previo a ello fue necesario diseñar y rediseñar estrategias de apoyo, éstas se valoraron positivamente, reconociendo el trabajo y compromiso de docentes que se involucraron en planificar e implementar formas alternativas de enseñanza.

Aparece como una dimensión relevante las características y metodologías de enseñanza aprendizaje y sistemas de apoyo disponibles en la ES, las que pueden facilitar o dificultar la experiencia académica de EcD. Se reconoció que el apoyo de un Programa como PIANE es significativo al momento de implementar apoyos y adecuaciones curriculares, las que podrán ser generales o específicas según área disciplinar. Se atribuye a la universidad el rol de generar adaptaciones que desafíen los mecanismos de egreso y perfil profesional, y las formas tradicionales de enseñanza, mediante apoyo no solo a estudiantes sino también a docentes a fin de que puedan conocer metodologías diversas y entender el sentido de las adecuaciones como elementos de equidad y no como condiciones de ventaja.

$$
\begin{aligned}
& \text {...tenemos mucha dificultad con los profesores que tienen esta sensación...de no estar } \\
& \text { generando más ayuda... (111, APC-7) }
\end{aligned}
$$

Del mismo modo en que parece relevante la promoción de ajustes desde organismos centrales de la institución, se releva la importancia de avanzar en el diseño de estrategias más específicas de apoyo, teniendo en consideración que las trayectorias de EcD sensorial en muchos casos serán distintas a las tradicionales y probablemente tomarán más tiempo. En este sentido, desde el grupo de APC se plantea la importancia de pensar en la graduación efectiva más que oportuna, refiriéndose a la mayor valoración de aprendizaje por sobre cursar la carrera en el tiempo determinado de manera estándar, situación que además se suma a la carga de complementar el estudio con apoyos académicos es espacios distintos a las clases.

...tienen una trayectoria distinta, eh, se consideran a veces quizás cursos de nivelación que están como fuera del plan curricular usual, entonces obviamente extiende los plazos...ni siquiera tiene como sentido hablar de titulación exacta, si no que...de titulación efectiva. (80, APC-5)

...hay un tras bambalinas...de los estudiantes con necesidades educativas especiales, principalmente en el tema sensorial, que tiene que ver con todos los apoyos extra o los soportes que este estudiante tiene y también forman parte de su carga académica...y que hacen a lo mejor que este estudiante necesite o requiera tomar menos ramos para poder destinar también ese tiempo. (84, APC-6) 
Surgen tres elementos específicos que, desde la perspectiva de los participantes, son centrales respecto a las estrategias necesarias de desarrollar institucionalmente. El primero de ellos es el diseño de procesos diagnósticos que puedan contemplar dimensiones particulares de cada grupo y que sean complementarias a los instrumentos aplicados desde las distintas áreas disciplinarias, los cuales se centran en evaluar conocimientos al momento del ingreso.

$$
\begin{aligned}
& \text {...hay que hacer un diagnóstico bien específico para cada uno de los estudiantes, } \\
& \text { porque cada uno tiene necesidades que no son estándar. (105, APC-3) } \\
& \text { (los diagnósticos existentes) ...son súper estándar...son en áreas como muy básicas, yo } \\
& \text { creo que no hay un diagnóstico específico. (100, APC-4) }
\end{aligned}
$$

Estos procesos diagnósticos llevarían a definir: (a) trayectorias distintas a las establecidas y, (b) apoyos para la nivelación de competencias y/o contenidos específicos. La articulación entre ambos elementos resultaría en trayectorias de nivelación, según la denominación establecida por los participantes.

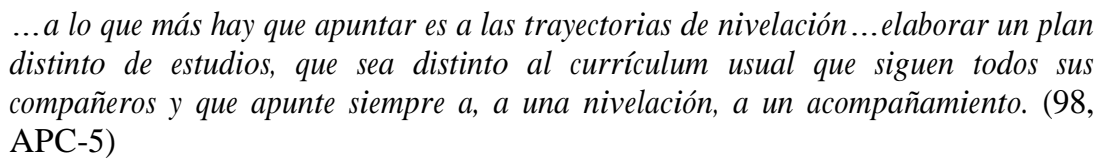

Respecto a las trayectorias, se consideró relevante que estas se enfoquen en el aprendizaje y deberían ser definidas considerando la reducción de carga académica pero también la planificación de la progresión curricular. Se valoraba positivamente la opción de flexibilizar la malla para partir con cursos de carácter más introductorio o teórico en aquellos casos en que la barrera se asocia a actividades prácticas. Esto favorece no solo el aprendizaje sino también la percepción del estudiante sobre su posibilidad de avance curricular.

$$
\begin{aligned}
& \text {...los alumnos van tomando menos carga y con eso los ayudamos a avanzar muy bien } \\
& \text { académicamente, pero también, se frustran con menos facilidad y van adquiriendo } \\
& \text { mayor seguridad en sí mismos. (111, APC-7) }
\end{aligned}
$$

En relación con los apoyos para la nivelación y/o desarrollo de competencias, se plantean como estrategias que podrían reemplazar las estrategias institucionales existentes o bien complementarlas. Esto debido a que se presentan necesidades de acceso a la información específicas o brechas académicas poco típicas y, en ocasiones, asociadas solo a ciertas áreas.

\subsubsection{Análisis relacional}

Como se observa en la Figura 2, existen distintas variables que interactúan en la experiencia académica de EcD sensorial en ES. Se identifican características de la escolaridad a nivel nacional, en donde los ajustes o apoyos se perciben escasos o insuficientes. Desde la ES, y los desafíos que ésta supone para cualquier estudiante, fue posible identificar desafíos específicos para EcD, según el tipo y grado de discapacidad en que esta se presenta, que parecen estar influidos además por su experiencia escolar. La posibilidad de contar con trayectorias escolares más enriquecidas, y con apoyos dentro o fuera de la escuela, o de haber accedido a la enseñanza de herramientas para la accesibilidad, situaría en un mejor escenario académico a los EcD sensorial. 


\section{Figura 2}

Modelo relacional. Permite una aproximación al fenómeno, dando cuenta de los principales elementos que interactúan en la experiencia académica de tfcD sensorial en tfS

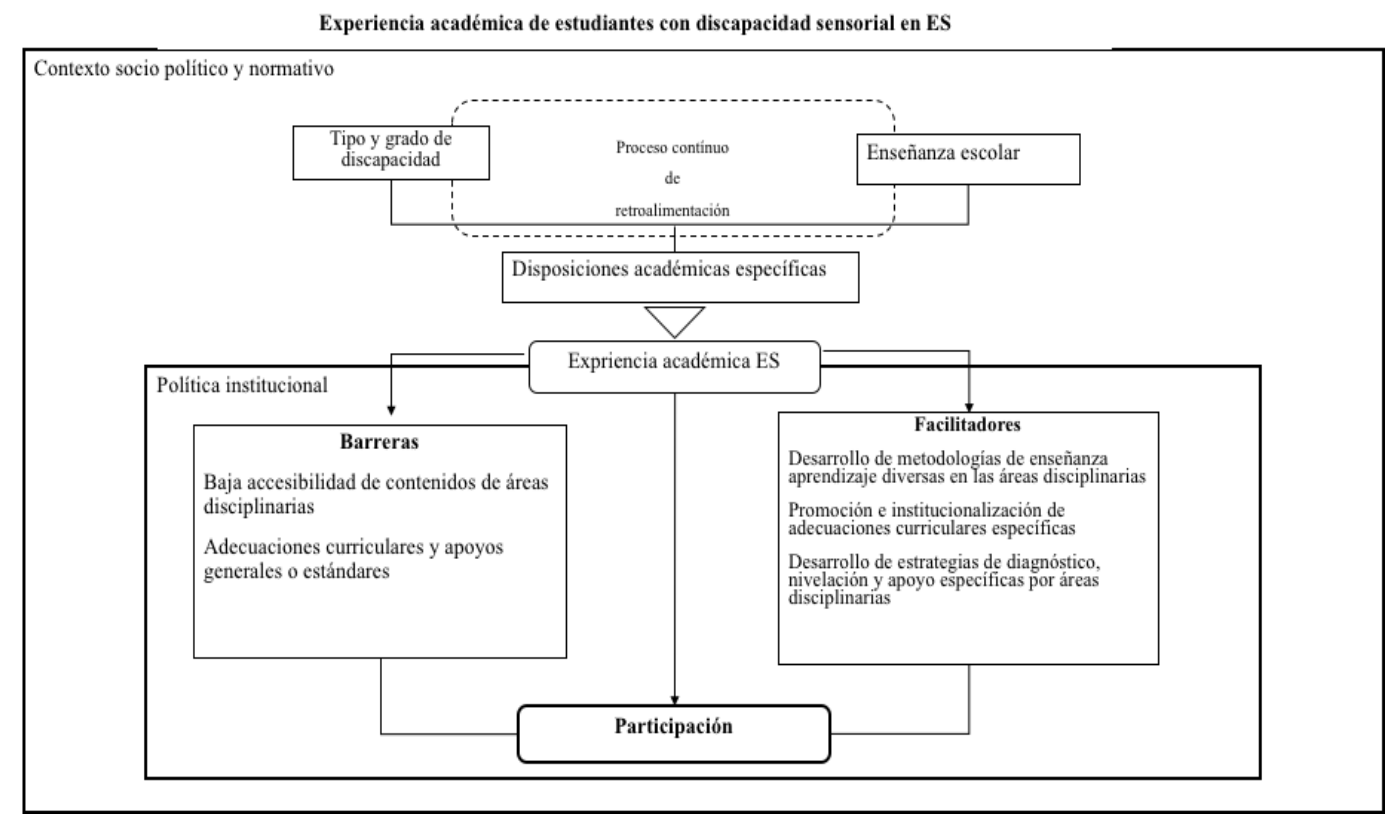

Lo antes mencionado no se traduce en la inexistencia de brechas académicas específicas, sino en mayor o menor proximidad a los contenidos que se esperan alcanzar en la ES. Estas brechas se dejarían ver en los contenidos, pero también en las estrategias disponibles para acceder a ellos, las que pueden tener que ver con aspectos desde el uso de una tecnología hasta la comprensión de conceptos abstractos relevantes para distintas áreas disciplinarias. Cabe destacar que la profundidad y amplitud de estas brechas serían mayores para estudiantes sordos debido a las características del acceso a conceptualizaciones de este tipo.

De este modo, la experiencia académica de EcD sensorial parte desde la disposición generada por las características de esta y las opciones que tuvieron de acceder a soportes o apoyos pertinentes durante su escolaridad. Lo anterior interactúa con aspectos contextuales y característicos de la ES que pueden desfavorecer el aprendizaje de estudiantes con mayor énfasis en áreas disciplinarias como ciencias y matemáticas, específicamente por las metodologías disponibles para su enseñanza, en donde se percibe más complejo incorporar ajustes o diversificar los elementos que componen el programa de los cursos.

En caso de ser posible implementar adecuaciones y apoyos para EcD sensorial, estas dependen en gran medida de la existencia de políticas institucionales que las promuevan y de la comprensión de su sentido por parte del cuerpo docente. Se considera que las adecuaciones y apoyos, en caso de que existan, pueden llegar a ser planteados de manera muy general, siendo inefectivos para la profundidad o amplitud de las brechas específicas descritas.

La predominancia de ciertas barreras varía en función de las condiciones que ofrece el contexto ante las disposiciones particulares que traen los estudiantes dada su historia escolar. Esto sugiere que la relación que se produce entre estas variables es dinámica y compleja, de manera que las barreras que enfrentan los estudiantes no deberán 
comprenderse de manera estereotipada según su tipo y grado de discapacidad como características que pertenecen a ellos, sino como tendencias que en su paso por la ES pueden manifestarse de manera diversa.

Desde lo anteriormente planteado, cuando la ES cuenta o tiene el potencial de desarrollar mecanismos específicos de ajuste y apoyo, el estudiantado tendrá una experiencia universitaria más enriquecida y positiva. Para alcanzar esta meta se visualizan tres grandes elementos, en primer lugar, el contar con apoyos institucionales para el desarrollo de metodologías de enseñanza aprendizaje diversa, especialmente en las áreas disciplinarias descritas como más complejas; en segundo lugar, la promoción e institucionalización de adecuaciones curriculares específicas, y en este sentido, considerar el análisis de la progresión de asignaturas, más que la sola reducción de éstas durante la trayectoria universitaria, como estrategia eficaz para alcanzar mejores aprendizajes; $y$, en tercer lugar, el desarrollo de procesos diagnósticos que permitan definir apoyos o nivelaciones específicas que sean reconocidos institucionalmente, por ejemplo, desde la asignación de créditos (asignaturas cursadas), dado el trabajo que significa participar de estas intervenciones. Finalmente, estas acciones buscarían enriquecer el paso al aprendizaje universitario, las cuales deberán encontrar sustento en el establecimiento de normativas internas asociadas al diseño, ejecución y evaluación de estrategias focalizadas de apoyo y ajuste curricular.

\section{Discusión y conclusiones}

En las investigaciones sobre discapacidad predominan metodologías cualitativas (PazMaldonado, 2020) que abordan la experiencia desde el estudiantado y la docencia, encontrándose escasos análisis estadísticos (Ocampo, 2018) o desde la mirada de actores institucionales en otros roles que las enriquezcan. Por ello este estudio buscó profundizar en ello mediante la caracterización de la participación académica de $\mathrm{EcD}$ sensorial en la UC, a través del análisis de datos institucionales que fueron complementados con la percepción de actores clave que trabajan en distintas áreas disciplinarias, apoyo académico específico y acompañamiento estudiantil, a fin de describir, desde esta perspectiva, barreras para la participación educativa en general, considerando la etapa escolar, y los posibles desarrollos o iniciativas que podrían aportar las IES para favorecer dicha participación.

Los resultados mostraron que la participación de EcD sensorial en las distintas áreas disciplinarias analizadas es equivalente al 1\%, cifra similar a la presente en Latinoamérica y Europa respecto de EcD. Los participantes del estudio en roles de apoyo o acompañamiento estudiantil valoraron negativamente esta cifra, reconociendo que, pese al desarrollo de políticas en esta línea, la participación de EcD en ES sigue siendo escasa a nivel nacional.

Fue posible identificar barreras para la participación que varían según tipo de discapacidad, siendo más amplias y profundas en quienes presentan discapacidad auditiva, especialmente en estudiantes sordos que se comunican mediante LSCh. La tendencia respecto al uso de apoyos evidenció que la mayoría es de tipo académico, con foco en la nivelación de contenidos y desarrollo de habilidades transversales. Esto sugiere que los ajustes centrados solo en accesibilidad no resuelven todas las dificultades que enfrentan los EcD sensorial en ES. Sumado a ello, los resultados muestran que EcD auditiva usan 
apoyos solo académicos en menor proporción que EcD visual, quienes, a su vez, usan en menor proporción apoyos solo de accesibilidad.

Lo anterior muestra que los desafíos que enfrentan EcD sensorial en ES tienen que ver también con la posibilidad de aprender contenidos durante la etapa escolar, la cual se configura como una importante variable en la comprensión de brechas específicas, especialmente en el área de CNM, lo que concuerda con investigaciones que demuestran su menor participación en carreras científicas dadas las barreras de acceso (Eickmeyer et al., 2012; Milic Babic y Dowling, 2017; Mosia y Phasha, 2017), reconociéndose en esta área menores conocimientos sobre metodologías alternativas de enseñanza.

En esta línea, la implementación de adecuaciones curriculares y apoyos académicos típicos o estandarizados no sería suficiente para cubrir las necesidades particulares de EcD sensorial que no pudieron contar con ajustes durante la etapa escolar. Esto coincide con evidencias que sostienen que, pese a los avances en inclusión escolar y los esfuerzos realizados por los equipos que componen los establecimientos, aún existen importantes desafíos (López et al., 2014), especialmente en la articulación y nexos entre la etapa secundaria y la ES y en cómo ambas pueden potenciarse para responder a las necesidades de manera específica. En este sentido, la creación de los PIE ha significado un aporte para la participación de $\mathrm{EcD}$, sin embargo, dadas algunas condiciones de esta estrategia, no ha sido posible responder a la diversidad de necesidades que presenta el estudiantado. Así, por ejemplo, es posible ver que los recursos económicos o humanos no siempre son suficientes para dar una respuesta efectiva a las necesidades que se presentan (Tamayo, et al. 2018). De este modo, la especificidad de las brechas de EcD sensorial releva la importancia de un abordaje mayor desde la política pública a fin de fortalecer los esfuerzos que realiza el mundo escolar, comprendiendo que la enseñanza de $\mathrm{PcD}$ en su globalidad requiere de consideraciones específicas sobre los soportes académicos mínimos en cada una de las etapas educativas, siendo necesarias estrategias de tránsito efectivas y pertinentes a la ES.

Lo antes descrito, y el incremento de EcD a la ES, plantea desafíos para las instituciones, quienes han debido reflexionar y aprender a diseñar e implementar estrategias de apoyo que hace algunas décadas atrás no se visualizaban como parte de su quehacer. Es por ello que los resultados evidencian una positiva valoración de estrategias institucionales específicas que promuevan la inclusión de $\mathrm{EcD}$ en la comunidad universitaria, especialmente en la planta docente. En particular, en este estudio se identificó que los elementos que facilitan la participación académica de $\mathrm{EcD}$ son: la existencia de políticas y lineamientos internos que permitan la institucionalización de adecuaciones curriculares, dentro de las que destacan la posibilidad de establecer trayectorias diversas centradas en el aprendizaje más que en la graduación oportuna; y complementar las estrategias de apoyo estudiantil estandarizadas con el desarrollo de estrategias de diagnóstico, nivelación y apoyo, basadas en la identificación de dimensiones específicas por tipo de discapacidad y área disciplinar. Incorporar trayectorias de nivelación se presenta como una posible intervención o estrategia en niveles de ES que permitan no solo nivelar contenidos del currículum sino adquirir herramientas específicas necesarias para acceder a ellos. Esto implicaría que favorecer la inclusión en este nivel educativo, además de favorecer la accesibilidad, contemple acciones y ajustes centrados en la interacción generada entre las características del estudiantado con discapacidad y las áreas disciplinarias, siendo necesario avanzar hacia el diseño de estrategias focalizadas. 
Desde la revisión de literatura fue posible observar que, en países que cuentan con normativas que permiten asegurar apoyos desde la etapa escolar, los ajustes se promueven de modo más estandarizado en elementos de los cursos, como metodologías de clases, actividades y evaluaciones, siendo los programas de apoyo para EcD quienes se encargan de su indicación y gestión (The Association on Higher Education and Disability, 2012). Las características nacionales en torno al tema suponen la necesidad de considerar adecuaciones curriculares en un sentido mucho más amplio, y la presencia de estrategias de apoyo y acompañamiento complementarias que apunten al trabajo de elementos específicos en ES, tales como nivelación mediante metodologías accesibles, desarrollo de habilidades o competencias particulares, entre otras. Esto plantea importantes desafíos para la política pública, ya sea por medio del fortalecimiento de estrategias implementadas a nivel escolar y/o a través de la destinación de fondos estatales que permitan la sustentabilidad de soportes específicos durante toda la trayectoria académica de los estudiantes. En este sentido, es una valiosa señal la reciente instalación de la unidad de inclusión en la subsecretaría de educación superior del Ministerio de Educación.

Una limitación de este estudio es la reducida cantidad de EcD en la ES, y por consiguiente en la UC, no siendo posible realizar análisis estadísticos de carácter inferencial. Por otro lado, hubo dificultades para alcanzar mayor participación de quienes han brindado apoyo académico a EcD sensorial, siendo un grupo, probablemente, subrepresentado. Si bien se realizó el ejercicio de analizar relacionalmente las categorías emergentes con el objetivo de aportar una propuesta de ordenamiento de las categorías, en futuros estudios será necesario ampliar la muestra para alcanzar mayor saturación teórica.

El estudio consideró la percepción de actores clave institucionales, por lo que futuras investigaciones podrían enriquecer los hallazgos con la visión de los propios estudiantes. Así mismo, se abren preguntas de investigación asociadas a caracterizar las trayectorias escolares según tipo de discapacidad en interacción con distintas modalidades de enseñanza (p.e. regular, especial, regular con PIE), así como la identificación de diferencias individuales que puedan contribuir a comprender los factores que explicarían diferencias en las trayectorias académicas más allá de lo que el entorno escolar o universitario ofrece, lo anterior con el objetivo de fortalecer la conexión entre la educación escolar y ES, y ampliar las oportunidades de más EcD en la ES.

\section{Referencias}

Abu-Hamour, B. (2013). Faculty attitudes toward students with disabilities in a public university in Jordan. International tfducation Studies, 6(12), 74-81. https://doi.org/10.5539/ies.v6n12p74

Adams, M. y Holland, S. (2006). Improving access to higher education for disabled people. En M. Adams y S. Brown (Eds.), Towards inclusive learning in higher education (pp. 28-40). Routledge.

Agencia de Calidad de la Educación. (2019). Informe resultados nacionales estudiantes en situación de discapacidad sensorial $8^{\circ}$ básico 2019. Agencia de Calidad de la Educación.

Ainscow, M. y Miles, S. (2008). Por una educación para todos que sea inclusiva: ¿Hacia dónde vamos ahora? Perspectivas, 38, 17-44. 
Bellman, S., Burgstahler, S. y Hinke, P. (2015). Academic coaching: Outcomes from a pilot group of postsecondary STEM students with disabilities. Journal of Postsecondary tfducation and Disability, 28(1), 103-108.

Biggeri, M., Di Masi, D. y Bellacicco, R. (2019) Disability and higher education: assessing students' capabilities in two Italian universities using structured focus group discussions. Studies in Higher tfducation, 45(4) 909-924. https://doi.org/10.1080/03075079.2019.1654448

Booth, T. y Ainscow, M. (2000). Index for inclusion. Developing leaning and participation in schools. CSIE.

Borland, J. y James, S. (1999). The learning experience of students with disabilities in higher education. A case study of a UK university. Disability \& Society, 14(1), 85-101.

Carlberg, L. y Granlund, M. (2019). Achievement and participation in schools for young adolescents with self-reported neuropsychiatric disabilities: A cross-sectional study from the southern part of Sweden. Scandinavian Journal of Public Health, 47(2), 199-206. https://doi.org/10.1177/1403494818788415

Departamento de Evaluación, Medición y Registro Educacional. (2020). Informe de participación de personas en situación de discapacidad (PeSD) en el proceso de admisión 2020 de la prueba de selección universitaria (PSU). Actualización anual. DEMRE.

Díez, E. y Sánchez, S. (2015). Diseño universal para el aprendizaje como metodología docente para atender a la diversidad en la universidad. Aula Abierta, 43(2), 87-93. https://doi.org/10.1016/j.aula.2014.12.002

Domínguez, A. y Velasco, C. (s.f). La respuesta educativa a los estudiantes con discapacidad auditiva. Fundación Mapfre.

Eckes, S. y Ochoa, T. (2005). Students with disabilities: Transitioning from high school to higher education. American Secondary tfducation, 33(3), 6-20.

Eickmeyer, S., Do, K., Kirschner, K. y Curry, R. (2012). North American medical schools' experience with and approaches to the needs of students with physical and sensory disabilities. Academic Medicine: Journal of the Association of American Medical Colleges, 87(5), 567-573. https://doi.org/10.1097/ACM.0b013e31824dd129

Erbas, E. (2017). Strategies that teachers use to support the inclusion of students who are deaf and hard of hearing. Indiana University, Indiana.

Errandonea, M. (2016). Participación de personas en situación de discapacidad en el sistema de educación superior chileno. Repositorio Académico Universidad de Chile.

Fundación Universia. (2019). IV tfstudio sobre el grado de inclusión del sistema universitario español. Fundación Universia.

Hayes, C. (s.f). Teaching mathematics to students with hearing loss. UCONN.

Hewett, R., Douglas, G., McLinden, M. y Keil, S. (2017). Developing an inclusive learning environment for students with visual impairment in higher education: Progressive mutual accommodation and learner experiences in the United Kingdom. tfuropean Journal of Special Needs tfducation, 32(1), 89-109. https://doi.org/10.1080/08856257.2016.1254971

Hewett, R., Douglas, G., McLinden, M. y Keil, S. (2020). Balancing inclusive design, adjustments and personal agency: Progressive mutual accommodations and the experiences of university students with vision impairment in the United Kingdom. International Journal of Inclusive tfducation, 24(7), 754-770. https://doi.org/10.1080/13603116.2018.1492637

Higher Education Research Institute. (2011). College students with "hidden" disabilities: The freshman survey fall 2010. University of California. 
Humphrey, N., Wigelsworth, M., Barlow, A. y Squires, G. (2013). The role of school and individual differences in the academic attainment of learners with special educational needs and disabilities: A multi-level analysis. International Journal of Inclusive tfducation, 17(9), 909931. https://doi.org.pucdechile.idm.oclc.org/10.1080/13603116.2012.718373

Konur, O. (2006). Teaching disabled students in higher education. Teaching in Higher tfducation, 11(3), 351-363. https://doi.org/10.1080/13562510600680871

Lissi, M., Zuzulich, M., Hojas, A., Achiardi, C., Salinas, M. y Vásquez, A. (2013). tfn el camino hacia la educación superior inclusiva en Chile. Pontificia Universidad Católica de Chile.

Lombardi, A. Vukovic, B. y Sala-Bars, I. (2015) International comparisons of inclusive instruction among college faculty in Spain, Canada and United States. Journal of Postsecondary tfducation and Disability, 28(4), 447-460.

López, V., Julio, C., Morales, M., Rojas, C. y Pérez, M. (2014). Barreras culturales para la inclusión: Políticas y prácticas de integración en Chile. Revista de tfducación, 363, 256-281. https://doi.org/10.4438/1988-592X-RE-2012-363-180

Medrano, D. (2009). tfstudiantes universitarios con discapacidad: Análisis de los relatos de sus vivencias en el ámbito educativo Repositorio UC. https://doi.org/10.4438/1988-592X-RE-2012-363180

Mella, S., Díaz, N., Muñoz, S., Orrego, M. y Rivera, C. (2014). Percepción de facilitadores, barreras y necesidades de apoyo de estudiantes con discapacidad en la Universidad de Chile. Revista Latinoamericana de tfducación Inclusiva, 8(1), 63-80.

Milic Babic, M. y Dowling, M. (2017). Social support, the presence of barriers and ideas for the future from students with disabilities in the higher education system in Croatia. Disability \& Society, 30(4), 614-629. https://doi.org/10.1080/09687599.2015.1037949

McKinney, E. L. y Swartz, L. (2020). Integration into higher education: Experiences of disabled students in South Africa. Studies in Higher tfducation, 79, art 1. http://dx.doi.org/10.1080/03075079.2020.1750581

Moriña, A., Cortés, M. D. y Molina, V. M. (2015). What if we could imagine the ideal faculty? Proposals for improvement by university students with disabilities. Teaching and Teacher tfducation, 52, 91-98. https://doi.org/10.1016/j.tate.2015.09.008

Moriña, A., López-Gavira, R. y Molina, V. M. (2017). What if we could imagine an ideal university? Narratives by students with disabilities. International Journal of Disability, Development and tfducation, 64(4), 353-367.

https://doi-org.pucdechile.idm.oclc.org/10.1080/1034912X.2016.1228856

Mosia, P. A. y Phasha, N. (2017). Access to curriculum for students with disabilities at higher education institutions: how does the national university of Lesotho fare? African Journal of Disability, 6(1), 1-13. https://doi-org.pucdechile.idm.oclc.org/10.4102/ajod.v6i0.257

Ocampo, A. (2018). Sobre la condición tropológica de la educación inclusiva: Elementos para una nueva crítica educativa. Revista de tfducación Inclusiva, 2(2), 16-46. https://revista.celei.cl/index.php/PREI/article/view/254/195

Organización Mundial de la Salud (2011). Informe mundial sobre la discapacidad 2011. OMS.

Paz-Maldonado, E. (2020). Revisión sistémica: Inclusión educativa de estudiantes universitarios en situación de discapacidad en América Latina. tfstudios Pedagógicos, 44(1), 413-429. https://doi.org/10.4067/S0718-07052020000100413 
Rodríguez, A. y Álvarez, E. (2014). Estudiantes con discapacidad en la universidad. Un estudio sobre su inclusión. Revista de la Complutense, 25 (2), 457-479.

https://doi.org/10.5209/rev_RCED.2014.v25.n2.41683

Rodríguez, G. y Valenzuela, B. (2019) Acceso y permanencia de estudiantes con discapacidad en las universidades chilenas. Sinéctica, 53, 1-16. https://doi.org/10.31391/S2007-7033(2019)0053-002

Servicio de Información de Educación Superior. (2014). Panorama de la educación superior en Chile 2014. Ministerio de Educación.

Servicio Nacional de la Discapacidad. (2004). Primer estudio nacional de la discapacidad. Servicio Nacional de la Discapacidad.

Servicio Nacional de la Discapacidad. (2015). Segundo estudio nacional de la discapacidad. Servicio Nacional de la Discapacidad.

Salinas, M. , Lissi, M., Medrano, D., Zuzulich, M. y Hojas, A. (2013). La inclusión en la educación superior: desde la voz de estudiantes chilenos con discapacidad. Revista Ibero-americana de tfducação, 63, 77-98. https://doi.org/10.35362/rie630502

Sentenac, M., Lach, L. M., Gariepy, G. y Elgar, F. J. (2019). Education disparities in young people with and without neurodisabilities. Developmental Medicine \& Child Neurology, 61(2), 226231. https://doi-org.pucdechile.idm.oclc.org/10.1111/dmcn.14014

Tamayo, M., Besoaín, Á. y Rebolledo, J. (2018). Determinantes sociales de la salud y discapacidad: actualizando el modelo de determinación. Gaceta Sanitaria, 32, 96-100. httpS://doi.org/10.1016/j.gaceta.2016.12.004

The Association on Higher Education and Disability (2012). Supporting accommodation requests: Guidance on documentation practices. AHEAD.

Treviño, E., Villalobos, C., Hernández, C., Vielma, C. y Valenzuela, J. P. (2016). Trayectorias escolares de los estudiantes y agrupamiento al interior del aula en los colegios chilenos de enseñanza media. Análisis de la heterogeneidad académica al interior de las escuelas. Pensamiento tfducativo, 53(2), 1-17. https://doi-org.pucdechile.idm.oclc.org/10.7764/PEL.53.2.2016.5

Wessel, R. D., Jones, J. A., Markle, L. y Westfall, C. (2009). Retention and graduation of students with disabilities: Facilitating student success. Journal of Postsecondary tfducation and Disability, 21(3), 116-125.

Zuzulich, M. S., Achiardi, C., Hojas, A. M. y Lissi, M. R. (2014). Inclusión de estudiantes con discapacidad en universidades chilenas: mecanismos de admisión y recursos para su implementación. En Centro de Políticas Públicas UC (Eds.), Propuestas para Chile (pp. 5588). Pontificia Universidad Católica de Chile.

\section{Breve CV de los/as autores/as}

\section{Catalina García}

Psicóloga y Magíster en Psicología Educacional de la Pontificia Universidad Católica de Chile (UC). Especialista en Educación de Talentos de la Universidad de Nijmegen y la UC. Diplomada en Educación Inclusiva y Discapacidad UC; con Formación en Diseño Universal de Aprendizaje y Pasantía en Center for Applied Special Technology. Ha realizado docencia universitaria, investigación e intervención profesional, vinculadas con aprendizaje y evaluación en diversidad y equidad. Fue Gerente de Desarrollo en el Centro de Desarrollo de Tecnologías de Inclusión UC, y actualmente es Directora de Inclusión de la UC, a cargo de vías de admisión de equidad; inserción 
y acompañamiento estudiantil; diagnósticos, apoyos académicos y adecuación curricular; y monitoreo y alertas tempranas. ORCID ID: https://orcid.org/0000-0002-0564-4521. Email: cigarcia@uc.cl

\section{Javier Farías}

Sociólogo de la Pontificia Universidad Católica de Chile (UC). Magíster en Gestión y Políticas Públicas y Diplomado en Gerencia Pública de la Universidad de Chile. Ha realizado docencia universitaria, investigación y coordinación de programas vinculados con gestión y políticas públicas, evaluación y educación, equidad e inclusión. Se desempeñó como Jefe de Proyectos de Inclusión de la Escuela de Ingeniería UC y, actualmente, es docente del Departamento de Ingeniería Industrial de la Universidad de Chile y Profesional de Monitoreo y Estudios de la Dirección de Inclusión de la UC, colaborando en el seguimiento de las trayectorias académicas de estudiantes, sistema de alerta tempranas y apoyo y asesoría en procesos y evaluación de resultados. ORCID ID: https://orcid.org/0000-0002-4058-9302. Email: jnfarias@uc.cl

\section{Daniela Reyes}

Licenciada en Educación y Profesora de Educación Diferencial Especialista en Problemas Visuales de la Universidad Metropolitana de Ciencias de la Educación. Psicopedagoga, Diplomada en Problemas de Aprendizaje de la Universidad Central y Diplomada en Aprendizaje Profundo de la Pontificia Universidad Católica de Chile (UC). Se ha desempeñado como profesional de apoyo a la inclusión de estudiantes con necesidades educativas especiales en educación escolar y superior; además de labores de docencia en Educación Superior. En el Programa para la Inclusión de Alumnos con Necesidades Especiales de la UC realiza acompañamiento estudiantil y de unidades académicas, adecuación de material, tecnologías para la inclusión y formación de la comunidad universitaria. ORCID ID: https://orcid.org/0000-0003-1537-6601. Email: dreyesr@uc.cl

\section{Andrea Vásquez}

Psicóloga y Magíster en Psicología Educacional de la Pontificia Universidad Católica de Chile (UC). Diplomada en Educación Inclusiva y Discapacidad UC. Ha realizado docencia universitaria, proyectos de investigación, apoyo y guía de tesis y asesoría en temáticas de inclusión y discapacidad. Actualmente es la Coordinadora del Programa para la Inclusión de Alumnos con Necesidades Especiales de la UC, a cargo del acompañamiento, apoyo académico y de accesibilidad para estudiantes con discapacidad, definición e implementación de adecuaciones curriculares, orientación y asesoría a equipos docentes y unidades académicas. ORCID ID: https://orcid.org/0000-0001-8616-5559. Email: alvasque@uc.cl 